\title{
Automated Amortised Resource Analysis for Term Rewrite Systems
}

\author{
Georg Moser ${ }^{\mathrm{a}}$, Manuel Schneckenreither ${ }^{\mathrm{b}}$ \\ a Department of Computer Science, University of Innsbruck, Austria, \\ ${ }^{b}$ Department of Information Systems, Production and Logistics Management, University of \\ Innsbruck, Austria,
}

\begin{abstract}
In this paper we establish an automated amortised resource analysis for term rewrite systems. The method is presented in an annotated type system and gives rise to polynomial bounds on the innermost runtime complexity of the analysed term rewrite system. Our analysis does not restrict the input rewrite system in any way so that rewrite systems may serve as abstractions of first-order, eagerly evaluated functional programs over user-defined inductive data-types. This facilitates integration in a general framework for resource analysis of programs. In particular, we have implemented the method and integrated it into our analysis tool $\mathrm{T}_{\mathrm{C}}^{\mathrm{T}}$. Furthermore, we have coupled the established analysis with a complexity reflecting transformation from pure OCaml programs. This extends the provided analysis to a fully automated resource analysis of higher-order functional programs.
\end{abstract}

Keywords: analysis of algorithms, amortised complexity, functional programming, types, automation

\section{Introduction}

Amortised resource analysis [1, 2] is a powerful method to assess the overall complexity of a sequence of operations precisely. It has been established by Sleator and Tarjan in the context of self-balancing data structures, which sometimes require costly operations that however balance out in the long run.

For automated resource analysis, amortised cost analysis has been in particular pioneered by Hoffmann et al., whose RaML prototype has grown into a highly sophisticated analysis tool for higher-order functional programs, cf. 3]. In a similar spirit, resource analysis tools for imperative programs like COSTA [4], CoFloCo [5] and LOOPUS [6] have integrated amortised reasoning. In this paper, we establish a novel automated amortised resource analysis for term rewrite systems (TRSs for short).

Consider the rewrite system $\mathcal{R}_{1}$ in Figure 1 encoding a variant of an example by Okasaki [7, Section 5.2] (see also [8, Example 1]); $\mathcal{R}_{1}$ encodes an efficient implementation

Email addresses: georg.moser@uibk.ac.at (Georg Moser), manuel.schneckenreither@uibk.ac.at (Manuel Schneckenreither)

Preprint submitted to Elsevier

November 26, 2018 


$$
\begin{aligned}
& \text { 1: } \quad \operatorname{chk}(\text { que }(\text { nil }, r)) \rightarrow \text { que }(\operatorname{rev}(r), \text { nil }) \\
& \text { 7: } \quad \text { enq }(0) \rightarrow \text { que(nil, nil) } \\
& 2: \operatorname{chk}(\text { que }(x \sharp x s, r)) \rightarrow \text { que }(x \sharp x s, r) \\
& \text { 8: } \quad \operatorname{rev}^{\prime}(\text { nil, } y s) \rightarrow \text { ys } \\
& \text { 3: } \quad \operatorname{tl}(\text { que }(x \sharp f, r)) \rightarrow \operatorname{chk}(\text { que }(f, r)) \\
& \text { 9: } \\
& \operatorname{rev}(x s) \rightarrow \operatorname{rev}^{\prime}(x s, \text { nil }) \\
& \text { 4: } \operatorname{snoc}(q u e(f, r), x) \rightarrow \operatorname{chk}(q u e(f, x \sharp r)) \quad 10: \operatorname{hd}(\text { que }(x \sharp f, r)) \rightarrow x \\
& \text { 5: } \quad \operatorname{rev}^{\prime}(x \sharp x s, y s) \rightarrow \operatorname{rev}^{\prime}(x s, x \sharp y s) \\
& \text { 11: } \quad \text { hd }(\text { que }(\text { nil, } r)) \rightarrow \text { err_head } \\
& \text { 6: } \quad \operatorname{enq}(\mathrm{s}(n)) \rightarrow \operatorname{snoc}(\operatorname{enq}(n), n) \\
& \text { 12: } \quad \operatorname{tl}(\text { que }(\text { nil, } r)) \rightarrow \text { err_tail }
\end{aligned}
$$

Figure 1: Queues in Rewriting.

of a queue in functional programming. A queue is represented as a pair of two lists que $(f, r)$, encoding the initial part $f$ and the reversal of the remainder $r$. The invariant of the algorithm is that the first list never becomes empty, which is achieved by reversing $r$ if necessary. Should the invariant ever be violated, an exception (err_head or err_tail) is raised. To exemplify the physicist's method of amortised analysis [2] we assign to every queue que $(f, r)$ the length of $r$ as potential. Then the amortised cost for each operation is constant, as the costly reversal operation is only executed if the potential can pay for the operation. Thus, based on an amortised analysis, we may deduce the optimal linear runtime complexity for $\mathcal{R}$.

Taking inspirations from [8, 9], the amortised analysis is based on the potential method, as exemplified above. It employs the standard (small-step) semantics of innermost rewriting and exploits a footprint relation in order to facilitate the extension to TRSs. For the latter, we suit a corresponding notion of Avanzini et al. [10] to our context. Due to the small-step semantics we immediately obtain an analysis which does not presuppose termination. The incorporation of the footprint relations allows the immediate adaption of the proposed method to general rule-based languages. The most significant extension, however, is the extension to standard TRSs. TRSs form a universal model of computation that underlies much of declarative programming. In the context of functional programming, TRSs form a natural abstraction of strictly typed programming languages like RaML, but natively form foundations of non-strict languages and non-typed languages as well.

Our interest in an amortised analysis for TRSs is motivated by the use of TRSs as abstract program representation within our uniform resource analyse tool $T_{C} \mathrm{~T}[11]$. Rather than studying amortised cost analysis for a particular programming language, as provided for example by Hoffmann et al. in [3], we follow the general approach of static program analysis, where peculiarities of specific programming languages are suitable abstracted to give way to more general constructions like recurrence relations, cost relations, transition systems, term rewrite systems, etc.

Thus we first seek a more general discussion of amortised analysis in (first-order) TRSs. In this spirit we aim at an amortised resource for TRSs in its standard form: neither typed, not necessarily left-linear, confluent, nor constructor-based. Secondly, we extend the established resource analysis through a transformational approach, based on earlier work by Avanzini et al. [12, 13]. This provides us with a state-of-the-art tool for the resource analysis of pure OCaml programs, but more generally allows the analysis of 
general programs. Technically, the main contributions of the paper are as follows.

- Employing the standard rewriting semantics in the context of amortised resource analysis. This standardises the results and simplifies the presentations contrasted to related results on amortised analysis of TRSs cf. [8, 14]. We emphasise that our analysis does not presuppose termination.

- We overcome earlier restrictions to typed, completely defined, orthogonal and constructor TRSs. Thus, we establish an amortised analysis for standard first-order rewrite system, that is, the only restrictions required are the standard restrictions that (i) the left-hand side of a rule must not be a variable and (ii) no extra variables are introduced in rules.

- The analysis is lifted to relative rewriting, that is, the runtime complexity of a relative TRS $\mathcal{R} / \mathcal{S}$ is measured by the number of rule applications from $\mathcal{R}$, only. This extension is mainly of practical relevance, as required to obtain an automation of significant strength.

- The analysis has been implemented and integrated into $\mathrm{T}_{\mathrm{C}} \mathrm{T}$. We have assessed the viability of the method in context of the Termination Problem Database (TPDB) 1 as well as on an independent benchmark of TRSs.

- Finally, the aforementioned analysis has been coupled with the complexity reflecting HOCA transformation [12, 13] to provide a fully automated resource analysis of pure OCaml. This is based on a refined transformation taking types into account to improve the applicabilities of heuristics in the amortised analysis. We provide experimental evidence of the viability of the analysis.

This paper is structured as follows. In the next section we provide a high-level introduction into the method, exemplifying its use in the analysis of $B^{\star}$-trees. We believe this example to be of independent interest as it constitutes a challenge to other approaches in automated amortised analysis. In Section 3, we cover basics. In Section 4, we introduce the inference system and prove soundness of the method. In Section 5, we detail the implementation of the method and remark on challenges posed by automation. Section 6 provides the experimental assessment of the method. Finally, we conclude in Section 7. where we also sketch future work.

The paper is a revised and extended version of the original presentation of the established method in 15]. Apart from the usual correction of errors in the conference version, we have incorporated full formal details and additional examples. Furthermore, the extension to a resource analysis of higher-order function programs is new, which also triggered a renewed experimental evaluation.

\section{Worst-Case Analysis for Higher Order OCaml Programs}

Term rewrite systems form a generic computational model and are well suited for transformations from various program languages. Consequently our transformational

\footnotetext{
${ }^{1}$ See http://termination-portal.org/wiki/TPDB
} 
approach first transforms a given program into a TRS, whose resource consumption is later analysed (see [16]). Consider for instance the following program BTree, encoding lookup for a variant of $B^{\star}$-trees in OCaml. $B^{\star}$ trees are data structures usually used for indexing purposes in databases. The function lookup traverses through the given $B^{\star}$-tree while searching for an element. The invariant of the tree is, that each node consists of $n+1$ sub-trees, which are ordered and split among $n$ values. The data resides in the leaves of the tree. The actual number $n$ specifying the amount of sub-trees is usually set to a value such that via one disk read a full node including the separation criteria and links to the sub-trees is retrieved. Thus $n$ is related to the underlying hardware and therefore kept variable to be able to minimises the number of disk reads.

The worst-case runtime complexity of the program is linear in the size of the input tree, whereas the best-case complexity is constant due to the exceptions being raised for an invalid tree input. In case only valid tree inputs are considered the best-case complexity is also linear. Therefore once dropping lines 11 and 15, which is a reasonable decision under the assumption that an encapsulated inserting mechanism ensures to generate valid trees only, the worst-case and best-case complexities coincide. To the best of our knowledge our tool is currently the only one that that can assess the worst-case and best-case complexity of the example fully automatically. (See 14] for the amortised analysis for best-case complexity of TRSs.)

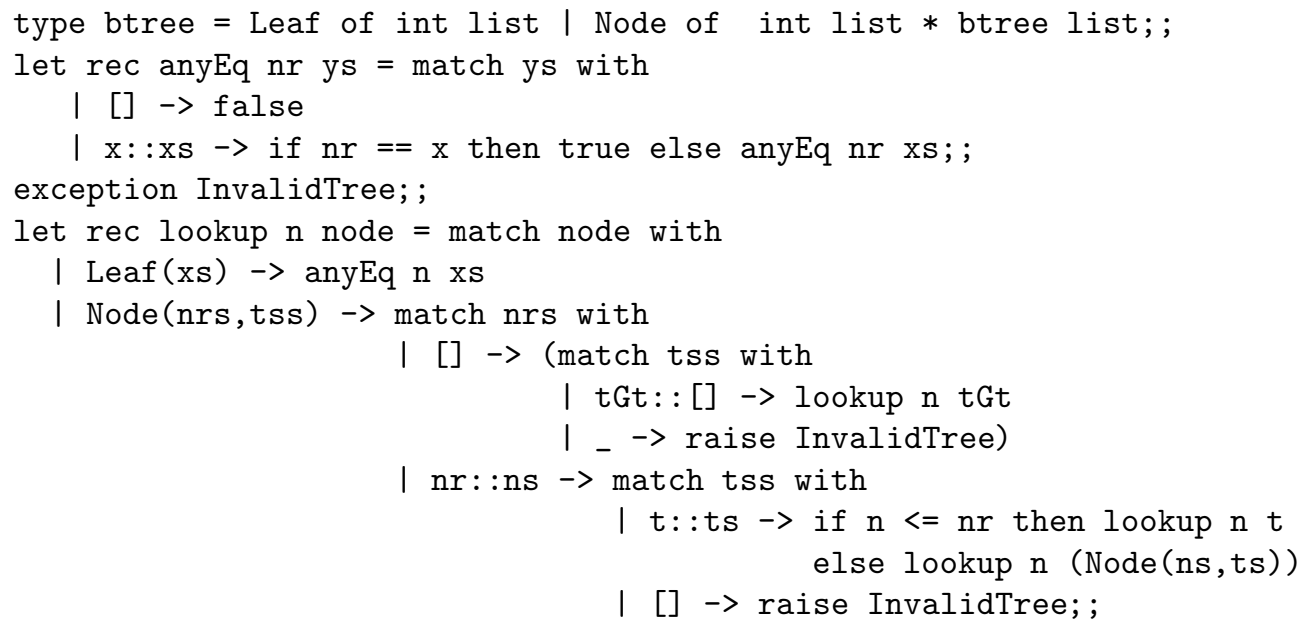

Exploiting the complexity preserving and complexity reflecting HOCA transformation due to Avanzini et al. [12, 13], we transform the OCaml program into an equivalent TRS $\mathcal{R}_{\text {btree }}$. The transformation employs defunctionalisation of the higher-order program and applies transformations like inlining, uncurrying and dead code elimination. The output of the transformation is a TRS with corresponding type information. The latter may be dropped, if we seek a standard TRS. Crucially the fully automatic transformation is complexity reflecting, that is, the complexity bounds of the obtained TRS $\mathcal{R}_{\text {btree }}$ reflects the runtime complexity of the above OCaml program, so that we can employ the downstream resource analysis of the rewrite system $\mathcal{R}_{\text {btree }}$ to bound the runtime complexity of the functional program. The resulting TRS $\mathcal{R}_{\text {btree }}$ (without type information) is given below. 


$$
\begin{aligned}
\operatorname{anyEq}(n r, \operatorname{nil}) & \rightarrow \text { false } \\
\operatorname{anyEq}(n r, x \sharp x s) & \rightarrow \operatorname{ite}(n r=x, \operatorname{true}, \operatorname{anyEq}(n r, x s)) \\
\operatorname{lookup}(n, \operatorname{Leaf}(x s)) & \rightarrow \operatorname{anyEq}(n, x s) \\
\operatorname{lookup}(n, \operatorname{Node}(\operatorname{nil}, t G t \sharp \operatorname{nil})) & \rightarrow \operatorname{lookup}(n, t G t) \\
\text { lookup }(n, \operatorname{Node}(n r \sharp n s, t \sharp t s)) & \rightarrow \operatorname{ite}(n \leq n r, \operatorname{lookup}(n, t), \operatorname{lookup}(n, \operatorname{Node}(n s, t s))) \\
\text { lookup }(n, \operatorname{Node}(\text { nil, nil })) & \rightarrow \perp \\
\text { lookup }(n, \operatorname{Node}(\operatorname{nil}, t \sharp(t 1 \sharp t s))) & \rightarrow \perp \\
\text { lookup }(n, \operatorname{Node}(t \sharp t s, \text { nil })) & \rightarrow \perp
\end{aligned}
$$

However, the current version of the HOCA prototype relaxes the exception handling and thus provides the fourth rule as lookup $(n, \operatorname{Node}($ nil, $t G t \sharp \mathbf{t s})) \rightarrow \operatorname{lookup}(n, t G t)$ and drops the second-to-last rule which becomes unnecessary. Note that this semantic change has no effect on the worst-case upper bound. Additionally the tool is unable to type the input program polymorphically. Thus constructors with different type contexts have to be renamed apart. We will be concerned with these issues in the future, but these are out of scope of this work.

The subsequent analysis of the obtained TRS is based on the amortised analysis established in the paper. This univariate amortised analysis is based on the potential method which is coached in syntax-directed annotated type system, where the function arguments and the function result are annotated with resource information, see Section 4 . If the TRS turns out to be resource bounded, that is, it is derivable in the inference system, then the differences in the potential functions before and after the evaluation provide the worst-case bounds. This is a consequence of the Soundness Theorem, Theorem 5 . Furthermore we provide suitable constrains on the resource annotations to allow the deduction of polynomial innermost runtime complexities, cf. Theorem 7 .

The construction is easy to mechanise as the proof search can be encoded into (in general) non-linear constraints, which can be subsequently handled by an SMT solver embedded in our prototype implementation. The non-linearity of the generated constraints stems from the fact that the inductive data types are not restricted and no type information is required a priori, see Section 5. As this is costly, we incorporated further heuristics to obtain linear constraints, following Hoffmann et al. 17, 18, 3]. Although these often speed up the analysis (see Section [6), they require comprehensive type information as they for example utilize the position of the recursive type parameters. HOCA infers following types for the BTree example.

$$
\begin{aligned}
\text { bool } & :=\perp \mid \text { false } \mid \text { true } \\
\operatorname{list}(a) & :=\text { nil } \mid a \sharp \operatorname{list}(a) \\
\text { nat } & :=0 \mid \mathrm{s}(\text { nat }) \\
\operatorname{tree}(a) & :=\operatorname{leaf}(\operatorname{list}(a)) \mid \operatorname{node}(\operatorname{list}(a), \operatorname{treelist}(a)) \\
\operatorname{treelist}(a) & :=\operatorname{nil}_{t} \mid a \sharp_{t} \operatorname{treelist}(a)
\end{aligned}
$$


For instance in the case of the type list $(a)$ the heuristics will select a multiple of the length of the list as appropriate measure for the potential of the given data structure. Thus, each application of $\sharp$ will require a certain potential $x$ and the second parameter list $(a)$ of the list has to pass the potential on, while nil and the first parameter of $\sharp$ must be typed with potential 0 . This yields $x$ times the length of the list as potential for this data structure. Clearly in most cases this makes sense. However, in the context of user-defined data structures this heuristic may lead to infeasibility.

Nonetheless, as in the run of the BTree example from above, it is often the case that the input program is (strongly) typed. To exploit this information, we make use of a recent implementation of the HOCA transformation that retains the type information. For BTree however, the aforementioned heuristics are inapplicable. This is due to the user-defined data structure which when being processed from top to bottom alternates between nodes of the tree and the list of possible sub-trees. Hence RaML, which only relies on heuristics, reports an unsupported recursive type error, while our prototype implementation can derive the linear worst-case complexity of BTree in terms of size of the input tree fully automatically.

\section{Preliminaries}

We assume familiarity with term rewriting [19, 20] but briefly review basic concepts and notations.

Let $\mathcal{V}$ denote a countably infinite set of variables and $\mathcal{F}$ a signature, such that $\mathcal{F}$ contains at least one constant. The set of terms over $\mathcal{F}$ and $\mathcal{V}$ is denoted by $\mathcal{T}(\mathcal{F}, \mathcal{V})$. We write $\mathcal{V} \operatorname{ar}(t)$ to denote the set of variables occurring in term $t$. The size $|t|$ of a term is defined as the number of symbols in $t$.

We suppose $\mathcal{F}=\mathcal{C} \uplus \mathcal{D}$, where $\mathcal{C}$ denotes a finite, non-empty set of constructor symbols, $\mathcal{D}$ is a finite set of defined function symbols, and $\uplus$ denotes disjoint union. Defined function symbols are sometimes referred to as operators. A term $t$ is linear if every variable in $t$ occurs only once. A term $t^{\prime}$ is the linearisation of a non-linear term $t$ if the variables in $t$ are renamed apart such that $t^{\prime}$ becomes linear. The notion generalises to sequences of terms. A term $t=f\left(t_{1}, \ldots, t_{k}\right)$ is called basic, if $f$ is defined, and all $t_{i} \in \mathcal{T}(\mathcal{C}, \mathcal{V})$. We write $\operatorname{dom}(\sigma)(\operatorname{rg}(\sigma))$ to denote the domain (range) of $\sigma$.

Let $\rightarrow \subseteq S \times S$ be a binary relation. We denote by $\rightarrow^{+}$the transitive and by $\rightarrow^{*}$ the transitive and reflexive closure of $\rightarrow$. By $\rightarrow^{n}$ we denote the $n$-fold application of $\rightarrow$. If $t$ is in normal form with respect to $\rightarrow$, we write $s \rightarrow^{!} t$. We say that $\rightarrow$ is well-founded or terminating if there is no infinite sequence $s_{0} \rightarrow s_{1} \rightarrow \ldots$. It is finitely branching if the set $\{t \mid s \rightarrow t\}$ is finite for each $s \in S$. For two binary relations $\rightarrow_{A}$ and $\rightarrow_{B}$, the relation of $\rightarrow_{A}$ relative to $\rightarrow_{B}$ is defined by $\rightarrow_{A} / \rightarrow_{B}:=\rightarrow_{B}^{*} \cdot \rightarrow_{A} \cdot \rightarrow_{B}^{*}$.

A rewrite rule is a pair $l \rightarrow r$ of terms, such that (i) the root symbol of $l$ is defined, and (ii) $\mathcal{V} \operatorname{ar}(l) \supseteq \mathcal{V} \operatorname{ar}(r)$. A term rewrite system (TRS) over $\mathcal{F}$ is a finite set of rewrite rules. Observe that TRSs need not be constructor systems, that is, arguments of left-hand sides of rules may contain defined symbols. Such function symbols are called constructor-like, as below they will be sometimes subject to similar restrictions as constructor symbols.

The set of normal forms of a TRS $\mathcal{R}$ is denoted as $\operatorname{NF}(\mathcal{R})$, or NF for short. We call a substitution $\sigma$ normalised with respect to $\mathcal{R}$ if all terms in the range of $\sigma$ are ground normal forms of $\mathcal{R}$. Typically $\mathcal{R}$ is clear from context, so we simply speak of a normalised 
substitution. In the sequel we are concerned with innermost rewriting, that is, an eager evaluation strategy. Furthermore, we consider relative rewriting.

A TRS is left-linear if all rules are left-linear, it is non-overlapping if there a no critical pairs, that is, no ambiguity exists in applying rules. A TRS is orthogonal if it is left-linear and non-overlapping. A TRS is completely defined if all ground normalforms are values. Note that an orthogonal TRS is confluent. A TRS is constructor if all arguments of left-hand sides are basic.

The innermost rewrite relation $\stackrel{\mathrm{i}}{\rightarrow}_{\mathcal{R}}$ of a TRS $\mathcal{R}$ is defined on terms as follows: $s \stackrel{\mathrm{i}}{\rightarrow}_{\mathcal{R}} t$ if there exists a rewrite rule $l \rightarrow r \in \mathcal{R}$, a context $C$, and a substitution $\sigma$ such that $s=C[l \sigma], t=C[r \sigma]$, and all proper subterms of $l \sigma$ are normal forms of $\mathcal{R}$. In order to generalise the innermost rewriting relation to relative rewriting, we introduce the slightly technical construction of the restricted rewrite relation [21]. The restricted rewrite relation $\stackrel{\mathcal{Q}}{\rightarrow}_{\mathcal{R}}$ is the restriction of $\rightarrow_{\mathcal{R}}$ where all arguments of the redex are in normal form with respect to the TRS $\mathcal{Q}$. We define the innermost rewrite relation, dubbed $\stackrel{\mathrm{i}}{\rightarrow}_{\mathcal{R} / \mathcal{S}}$, of a relative TRS $\mathcal{R} / \mathcal{S}$ as follows.

$$
\stackrel{i}{\rightarrow}_{\mathcal{R} / \mathcal{S}}:=\stackrel{\mathcal{R} \cup \mathcal{S}}{\longrightarrow}_{\mathcal{S}}^{*} \cdot \stackrel{\mathcal{R} \cup \mathcal{S}}{\longrightarrow}_{\mathcal{R}} \cdot \stackrel{\mathcal{R} \cup \mathcal{S}}{\longrightarrow}_{\mathcal{S}}^{*} .
$$

Observe that $\stackrel{\mathrm{i}}{\rightarrow}_{\mathcal{R}}=\stackrel{\mathrm{i}}{\rightarrow}_{\mathcal{R} / \varnothing}$ holds.

Let $s$ and $t$ be terms, such that $t$ is in normal-form. Then a derivation $D: s \rightarrow_{\mathcal{R}}^{*} t$ with respect to a TRS $\mathcal{R}$ is a finite sequence of rewrite steps. The derivation height of a term $s$ with respect to a well-founded, finitely branching relation $\rightarrow$ is defined as $\operatorname{dh}(s, \rightarrow)=\max \left\{n \mid \exists t s \rightarrow^{n} t\right\}$.

Definition 1. We define the innermost runtime complexity (with respect to $\mathcal{R} / \mathcal{S}$ ): $\mathrm{rc}_{\mathcal{R}}(n):=\max \left\{\mathrm{dh}\left(t, \stackrel{\mathrm{i}}{\rightarrow}_{\mathcal{R} / \mathcal{S}}\right) \mid t\right.$ is basic and $\left.|t| \leqslant n\right\}$.

Intuitively the innermost runtime complexity wrt. $\mathcal{R} / \mathcal{S}$ counts the maximal number of eager evaluation steps in $\mathcal{R}$ in a derivation over $\mathcal{R} \cup \mathcal{S}$. In the definition, we tacitly assume that $\stackrel{\mathrm{i}}{\rightarrow}_{\mathcal{R} / \mathcal{S}}$ is terminating and finitely branching.

For the rest of the paper the relative TRS $\mathcal{R} / \mathcal{S}$ and its signature $\mathcal{F}$ are fixed. In the sequel of the paper, substitutions are assumed to be normalised with respect to $\mathcal{R} \cup \mathcal{S}$.

\section{Resource Annotations}

In this section, we establish a novel amortised resource analysis for TRSs. This analysis is based on the potential method and coached in an inference system. Firstly, we annotate the (untyped) signature by the prospective resource usage (Definition 2). Secondly, we define a suitable inference system, akin to a type system. Based on this inference system we delineate a class of resource bounded TRSs (Definition 6) for which we deduce polynomial bounds on the innermost runtime complexity for a suitably chosen class of annotations, cf. Theorem 7

A resource annotation $\vec{p}$ is a vector $\vec{p}=\left(p_{1}, \ldots, p_{k}\right)$ over non-negative rational numbers. The vector $\vec{p}$ is also simply called annotation. Resource annotations are denoted by $\vec{p}, \vec{q}, \vec{u}, \vec{v}, \ldots$, possibly extended by subscripts and we write $\mathcal{A}$ for the set of such annotations. For resource annotations $(p)$ of length 1 we write $p$. A resource annotation does not change its meaning if zeroes are appended at the end, so, conceptually, 
we can identify ( $)$ with (0) and also with 0 . If $\vec{p}=\left(p_{1}, \ldots, p_{k}\right)$ we set $k:=|\vec{p}|$ and $\max \vec{p}:=\max \left\{p_{i} \mid i=1, \ldots, k\right\}$. We define the notations $\vec{p} \leqslant \vec{q}$ and $\vec{p}+\vec{q}$ and $\lambda \vec{p}$ for $\lambda \geqslant 0$ component-wise, filling up with 0 s if needed. So, for example $(1,2) \leqslant(1,2,3)$ and $(1,2)+(3,4,5)=(4,6,5)$.

Definition 2. Let $f$ be a function symbol of arity $n$. We annotate the arguments and results of $f$ by resource annotations. A (resource) annotation for $f$, decorated with $k \in \mathbb{Q}^{+}$, is denoted as $\left[\overrightarrow{p_{1}} \times \cdots \times \overrightarrow{p_{n}}\right] \stackrel{k}{\rightarrow} \vec{q}$. The set of annotations is denoted $\mathcal{F}_{\text {pol }}$.

We lift signatures $\mathcal{F}$ to annotated signatures $\mathcal{F}: \mathcal{C} \cup \mathcal{D} \rightarrow\left(\mathcal{P}\left(\mathcal{F}_{\text {pol }}\right) \backslash \varnothing\right)$ by mapping a function symbol to a non-empty set of resource annotations. Hence for any function symbol we allow multiple types. In the context of operators this is also referred to as resource polymorphism. The inference system, presented below, mimics a type system, where the provided annotations play the role of types. If the annotation of a constructor or constructor-like symbol $f$ results in $\vec{q}$, there must only be exactly one declaration of the form $\left[\overrightarrow{p_{1}} \times \cdots \times \overrightarrow{p_{n}}\right] \stackrel{k}{\rightarrow} \vec{q}$ in $\mathcal{F}(f)$, that is, the annotation has to be unique. Moreover, annotations for constructor and constructor-like symbols $f$ must satisfy the superposition principle: If $f$ admits the annotations $\left[\overrightarrow{p_{1}} \times \cdots \times \overrightarrow{p_{n}}\right] \stackrel{k}{\rightarrow} \vec{q}$ and $\left[\overrightarrow{p_{1}^{\prime}} \times \cdots \times \overrightarrow{p_{n}^{\prime}}\right] \stackrel{k^{\prime}}{\rightarrow} \overrightarrow{q^{\prime}}$ then it also has the annotations $\left[\lambda \overrightarrow{p_{1}} \times \cdots \times \lambda \overrightarrow{p_{n}}\right] \stackrel{\lambda k}{\longrightarrow} \lambda \vec{q}\left(\lambda \in \mathbb{Q}^{+}\right.$, $\lambda \geqslant 0)$ and $\left[\overrightarrow{p_{1}}+\overrightarrow{p_{1}^{\prime}} \times \cdots \times \overrightarrow{p_{n}}+\overrightarrow{p_{n}^{\prime}}\right] \stackrel{k+k^{\prime}}{\longrightarrow} \vec{q}+\overrightarrow{q^{\prime}}$.

Example 1. To exemplify consider the sets $\mathcal{D}=\left\{\right.$ enq, rev, rev' ${ }^{\prime}$ snoc, chk, hd, tl $\}$ and $\mathcal{C}=$ $\{$ nil, $\sharp$, que, $0, s\}$, which together make up the signature $\mathcal{F}$ of the motivating example $\mathcal{R}_{1}$ in Figure 1. Annotations of the constructors nil and $\sharp$ would for example be as follows. $\mathcal{F}($ nil $)=\{[\stackrel{0}{\rightarrow} k \mid k \geqslant 0\}$ and $\mathcal{F}(\sharp)=\{[0 \times k] \stackrel{k}{\rightarrow} k \mid k \geqslant 0\}$. These annotations are unique and fulfill the superposition principle.

Note that, in view of superposition and uniqueness, the annotations of a given constructor or constructor-like symbol are uniquely determined once we fix the resource annotations for result annotations of the form $(0, \ldots, 0,1)$ (remember the implicit filling up with $0 \mathrm{~s}$ ). An annotated signature $\mathcal{F}$ is simply called signature, where we sometimes write $f:\left[\vec{p}_{1} \times \cdots \times \vec{p}_{n}\right] \stackrel{k}{\rightarrow} \vec{q}$ instead of $\left[\vec{p}_{1} \times \cdots \times \vec{p}_{n}\right] \stackrel{k}{\rightarrow} \vec{q} \in \mathcal{F}(f)$.

The next definition introduces the notion of the potential of a normal form. For rules $f\left(l_{1}, \ldots, l_{n}\right) \rightarrow r$ in non-constructor TRSs the left-hand side $f\left(l_{1}, \ldots, l_{n}\right)$ need not necessarily be basic terms. However, the arguments $l_{i}$ are deconstructed in the rule (app) that we will see in Figure 2. This deconstruction may free potential, which needs to be well-defined. This makes it necessary to treat defined function symbols in $l_{i}$ similar to constructors in the inference system (see Definition 5).

Definition 3. Let $v=f\left(v_{1}, \ldots, v_{n}\right)$ be a normal form and let $\vec{q}$ be a resource annotation. We define the potential of $v$ with respect to $\vec{q}$, written $\Phi(v: \vec{q})$ by cases. First suppose $v$ contains only constructors or constructor-like symbols. Then the potential is defined recursively.

$$
\Phi(v: \vec{q}):=k+\Phi\left(v_{1}: \vec{p}_{1}\right)+\cdots+\Phi\left(v_{n}: \vec{p}_{n}\right),
$$

where $\left[\vec{p}_{1} \times \cdots \times \vec{p}_{n}\right] \stackrel{k}{\rightarrow} \vec{q} \in \mathcal{F}(f)$. Otherwise, we set $\Phi(v: \vec{q}):=0$. 


$$
\begin{aligned}
& \frac{f \in \mathcal{C} \cup \mathcal{D} \quad\left[\vec{p}_{1} \times \cdots \times \vec{p}_{n}\right] \stackrel{k}{\rightarrow} \vec{q} \in \mathcal{F}(f)}{x_{1}: \vec{p}_{1}, \ldots, x_{n}:\left.\vec{p}_{n}\right|^{k} f\left(x_{1}, \ldots, x_{n}\right): \vec{q}}(\text { app }) \quad \frac{\left.\Gamma\right|^{k} t: \vec{q} \quad k^{\prime} \geqslant k}{\left.\Gamma\right|^{k^{\prime}} t: \vec{q}}\left(\mathrm{w}_{1}\right) \\
& \text { all } x_{i} \text { are fresh } \quad k=\sum_{i=0}^{n} k_{i}
\end{aligned}
$$

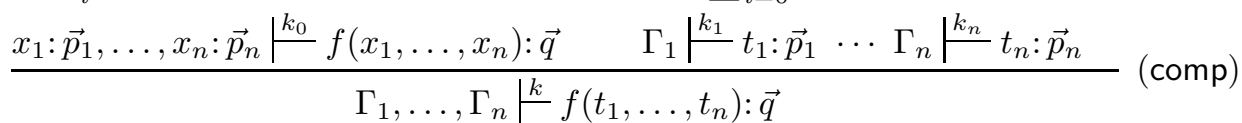

$$
\begin{aligned}
& \frac{\left.\Gamma\right|^{k} t: \vec{q}}{\Gamma, x:\left.\vec{p}\right|^{k} t: \vec{q}}\left(\mathrm{w}_{4}\right) \quad \frac{\Gamma, x: \vec{r}, y:\left.\vec{s}\right|^{k} t[x, y]: \vec{q} \quad \curlyvee(\vec{p} \mid \vec{r}, \vec{s}) \quad x, y \text { are fresh }}{\Gamma, z:\left.\vec{p}\right|^{k} t[z, z]: \vec{q}} \text { (share) } \\
& \frac{\Gamma, x:\left.\vec{r}\right|^{k} t: \vec{q} \quad \vec{p} \geqslant \vec{r}}{\Gamma, x:\left.\vec{p}\right|^{k} t: \vec{q}}\left(\mathrm{w}_{2}\right) \quad \frac{\left.\Gamma\right|^{k} t: \vec{s} \quad \vec{s} \geqslant \vec{q}}{\left.\Gamma\right|^{k} t:\left.\vec{q}\right|^{0} x: \vec{q}}\left(\mathrm{w}_{3}\right)
\end{aligned}
$$

Figure 2: Inference System for Term Rewrite Systems.

The sharing relation $\curlyvee\left(\vec{p} \mid \overrightarrow{p_{1}}, \overrightarrow{p_{2}}\right)$ holds if $\overrightarrow{p_{1}}+\overrightarrow{p_{2}}=\vec{p}$.

Lemma 1. Let $v$ be a a normal form. If $\curlyvee\left(\vec{p} \mid \overrightarrow{p_{1}}, \overrightarrow{p_{2}}\right)$ then $\Phi(v: \vec{p})=\Phi\left(v: \overrightarrow{p_{1}}\right)+\Phi\left(v: \overrightarrow{p_{2}}\right)$. Furthermore, if $\vec{p} \leqslant \vec{q}$ then $\Phi(v: \vec{p}) \leqslant \Phi(v: \vec{q})$.

Proof. The proof of the first claim is by induction on the structure of $v$. For constructor or constructor-like symbols the proof follows the pattern of Lemma 10 in [8]. We distinguish two cases. Either $v$ is only build from constructor symbols or constructorlike symbols. Then by superposition together with uniqueness the additivity property propagates to the argument types. For example, if we have the annotations $\mathrm{s}:[6] \stackrel{3}{\rightarrow} 12$, $\mathrm{s}:[10] \stackrel{5}{\rightarrow} 20$, and $\mathrm{s}:[x] \stackrel{8}{\rightarrow} y$ then we can conclude $x=16, y=22$, for this annotation must be present by superposition and there can only be one by uniqueness. Otherwise $v$ contains at least one $f \in \mathcal{D}$, but $f$ does not occur as argument of a left-hand side in $\mathcal{R}$. By definition $\Phi(v: \vec{q})=0$. Thus the lemma holds trivially. The second claim follows from the first one and non-negativity of potentials.

A (variable) context is a partial mapping from variables $\mathcal{V}$ to annotations. Contexts are denoted by upper-case Greek letters and depicted as sequences of pairs $x: \vec{q}$ of variables and annotations, where $x: \vec{q}$ in a variable context means that the resource $\vec{q}$ can be distributed over all occurrences of the variable $x$ in the term.

Definition 4. Our potential based amortised analysis is coached in an inference system whose rules are given in Figure 2. Let $t$ be a term and $\vec{q}$ a resource annotation. The inference system derives judgements of the form $\left.\Gamma\right|^{k} t: \vec{q}$, where $\Gamma$ is a variable context and $k \in \mathbb{Q}^{+}$denotes the amortised costs at least required to evaluate $t$.

Furthermore, we define a subset of the inference rules, free of weakening rules, dubbed the footprint of the judgement, denoted as $\Gamma \mid \frac{k}{\mathrm{fp}} t: \vec{q}$. For the footprint we only consider the inference rules (app), (comp), (share), and (var).

Occasionally we omit the amortised costs from both judgements using the notations $\Gamma \vdash t: \vec{q}$ and $\left.\Gamma\right|_{\mathrm{fp}} t: \vec{q}$. 
To ease the presentation we have omitted certain conditions, like the pairwise disjointedness of $\Gamma_{1}, \ldots, \Gamma_{n}$ in the rule (comp), that make the inference rules deterministic. However, the implementation (see Section 5) is deterministic, which removes redundancy in constraint building and thus improves performance. A substitution is called consistent with $\Gamma$ if for all $x \in \operatorname{dom}(\sigma)$ if $\Gamma \vdash x: \vec{q}$, then $\Gamma \vdash x \sigma: \vec{q}$. Recall that substitutions are assumed to be normalised. Let $\Gamma$ be a context and let $\sigma$ be a substitution consistent with $\Gamma$. Then $\Phi(\sigma: \Gamma):=\sum_{x \in \operatorname{dom}(\Gamma)} \Phi(x \sigma: \Gamma(x))$.

Definition 5. Let $f\left(l_{1}, \ldots, l_{n}\right) \rightarrow r, n \geqslant 1$, be a rule in the TRS $\mathcal{R} / \mathcal{S}$. Further suppose $f:\left[\vec{p}_{1} \times \cdots \times \vec{p}_{n}\right] \stackrel{k}{\rightarrow} \vec{q}$ is a resource annotation for $f$ and let $V:=\left\{y_{1}, \ldots, y_{m}\right\}$ denote the set of variables in the left-hand side of the rule. The potential freed by the rule is a pair consisting of a variable context $y_{1}: \vec{r}_{1}, \ldots, y_{m}: \vec{r}_{m}$ and an amortised cost $\ell$, defined as follows:

- The sequence $l_{1}^{\prime}, \ldots, l_{n}^{\prime}$ is a linearisation of $l_{1}, \ldots, l_{n}$. Set $Z:=\bigcup_{i=1}^{n} \mathcal{V} \operatorname{ar}\left(l_{i}^{\prime}\right)$ and let $Z=\left\{z_{1}, \ldots, z_{m^{\prime}}\right\}$, where $m^{\prime} \geqslant m$.

- There exist annotations $\vec{s}_{1}, \ldots, \vec{s}_{m^{\prime}}$ such that for all $i$ there exist costs $\ell_{i}$ such that $z_{1}: \vec{s}_{1}, \ldots, z_{m^{\prime}}: \vec{s}_{m^{\prime}} \mid \frac{\ell_{i}}{\mathrm{fp}} l_{i}^{\prime}: \vec{p}_{i}$.

- Let $y_{j} \in V$ and let $\left\{z_{j_{1}}, \ldots, z_{j_{o}}\right\} \subseteq Z$ be all renamings of $y_{j}$. Define annotations $\vec{r}_{j}:=\vec{s}_{j_{1}}+\cdots+\vec{s}_{j_{o}}$.

- Finally, $\ell:=\sum_{i=1}^{n} \ell_{i}$.

Example 2. Consider the rule enq $(\mathrm{s}(n)) \rightarrow \operatorname{snoc}(\operatorname{enq}(n), n)$ in the running example, together with the annotated signature enq: $[15] \stackrel{12}{\longrightarrow} 7$. The left-hand side contains the subterm $\mathrm{s}(n)$. Using the generic annotation $\mathrm{s}:[k] \stackrel{k}{\rightarrow} k$, the footprint $n: k \mid \frac{k}{\mathrm{fp}} \mathrm{s}(n): k$ is derivable for any $k \geqslant 0$. Thus, in particular the rule frees the context $n: 15$ and cost 15 .

Lemma 2. Let $f\left(l_{1}, \ldots, l_{n}\right) \rightarrow r \in \mathcal{R} / \mathcal{S}$ and let $c:\left[\vec{p}_{1} \times \cdots \times \vec{p}_{n}\right] \stackrel{0}{\rightarrow} \vec{q}$ denote a fresh, cost-free constructor. Let $y_{1}: \vec{r}_{1}, \ldots, y_{m}: \vec{r}_{m}$ and $\ell$ be freed by the rule. We obtain: $y_{1}: \vec{r}_{1}, \ldots, y_{m}: \vec{r}_{m} \mid \frac{\ell}{\mathrm{fp}} c\left(l_{1}, \ldots, l_{n}\right): \vec{q}$.

Proof. By assumption there exists a linearisation $l_{1}^{\prime}, \ldots, l_{n}^{\prime}$ of the arguments of the lefthand side of the rule. By definition no variable occurs twice in the sequence $l_{1}^{\prime}, \ldots, l_{n}^{\prime}$. Furthermore, for every $i=1, \ldots, n$, the following judgement is derivable:

$$
z_{1}: \vec{s}_{1}, \ldots, z_{m^{\prime}}: \vec{s}_{m} \mid \frac{\ell_{i}}{\mathrm{fp}} l_{i}^{\prime}: \vec{p}_{i}
$$

where $\ell=\sum_{i=1}^{n} \ell_{i}$. Observe that the definition of the annotations $\vec{r}_{j}$ embodies a repeated application of the sharing rule share. Thus in order to prove the lemma, it suffices to derive $z_{1}: \vec{s}_{1}, \ldots, z_{m^{\prime}}: \vec{s}_{m^{\prime}} \mid \frac{\ell}{f_{p}} c\left(l_{1}^{\prime}, \ldots, l_{n}^{\prime}\right): \vec{q}$. However, to derive the latter a single composition rule, together with the assumed derivations (10) suffices.

Based on Definition 5 we can now succinctly define resource boundedness of a TRS. The definition constitutes a non-trivial generalisation of Definition 11 in [8]. First the input TRS need no longer be sorted. Second the restriction on constructor TRSs has been dropped and finally, the definition has been extended to handle relative rewriting. 
Definition 6. Let $\mathcal{R} / \mathcal{S}$ be a relative TRS, let $\mathcal{F}$ be a signature and let $f \in \mathcal{F}$. An annotation $\left[\vec{p}_{1} \times \cdots \times \vec{p}_{n}\right] \stackrel{k}{\rightarrow} \vec{q} \in \mathcal{F}(f)$ is called resource bounded if for any rule $f\left(l_{1}, \ldots, l_{n}\right) \rightarrow$ $r \in \mathcal{R} \cup \mathcal{S}$, we have

$$
y_{1}: \vec{r}_{1}, \ldots, y_{l}:\left.\vec{r}_{l}\right|^{k+\ell-K^{\mathrm{rule}}} r: \vec{q},
$$

where $y_{1}: \vec{r}_{1}, \ldots, y_{l}: \vec{r}_{l}$ and $\ell$ are freed by the rule if $n \geqslant 1$ and $\ell=0$ otherwise. Here, the cost $K^{\text {rule }}$ for the application of the rule is defined as follows: (i) $K^{\text {rule }}:=1$ iff $f\left(l_{1}, \ldots, l_{n}\right) \rightarrow r \in \mathcal{R}$ and (ii) $K^{\text {rule }}:=0$ iff $f\left(l_{1}, \ldots, l_{n}\right) \rightarrow r \in \mathcal{S}$. We call an annotation cost-free resource bounded if the cost $K^{\text {rule }}$ is always set to zero.

A function symbol $f$ is called (cost-free) resource bounded if any resource annotation in $\mathcal{F}(f)$ is (cost-free) resource bounded. Finally, $\mathcal{R} / \mathcal{S}$ is called resource bounded, or simply bounded if any $f \in \mathcal{F}$ is resource bounded. Observe that boundedness of $\mathcal{R} / \mathcal{S}$ entails that the application of rules in the strict part $\mathcal{R}$ is counted, while the weak part $\mathcal{S}$ is not counted.

In a nutshell, the method works as follows: Suppose the judgement $\Gamma \mid \frac{k^{\prime}}{\mid} t \vec{q}$ is derivable and suppose $\sigma$ is consistent with $\Gamma$. The constant $k^{\prime}$ is an upper-bound to the amortised cost required for reducing $t$ to normal form. Below we will prove that the derivation height of $t \sigma$ (with respect to innermost rewriting) is bounded by the difference in the potential before and after the evaluation plus $k^{\prime}$. Thus if the sum of the potentials of the arguments of $t \sigma$ is in $\mathrm{O}\left(n^{k}\right)$, where $n$ is the size of the arguments and $k$ the maximal length of the resource annotations needed, then the innermost runtime complexity of $\mathcal{R} / \mathcal{S}$ lies in $\mathrm{O}\left(n^{k}\right)$.

More precisely consider the comp rule. First note that this rule is only applicable if $f\left(t_{1}, \ldots, t_{n}\right)$ is linear, which can always be obtained by the use of the sharing rule. Now the rule embodies that the amortised costs $k^{\prime}$ required to evaluate $t \sigma$ can be split into those costs $k_{i}^{\prime}(i \geqslant 1)$ required for the normalisation of the arguments and the $\operatorname{cost} k_{0}^{\prime}$ of the evaluation of the operator $f$. Furthermore the potential provided in the context $\Gamma_{1}, \ldots, \Gamma_{n}$ is suitably distributed. Finally the potential which remains after the evaluation of the arguments is made available for the evaluation of the operator $f$.

Before we proceed with the formal proof of this intuition, we exemplify the method on the running example.

Example 3 (continued from Example 1). $\mathrm{T}_{\mathrm{C}}^{\top}$ derives the following annotations for the operators in the running example.

$$
\begin{array}{ccc}
\text { enq : }[15] \stackrel{12}{\rightarrow} 7 & \text { rev }:[1] \stackrel{4}{\rightarrow} 0 & \text { rev }^{\prime}:[1 \times 0] \stackrel{2}{\rightarrow} 0 \\
\text { snoc: }:[7 \times 0] \stackrel{14}{\rightarrow} 7 & \text { hd : }[11] \stackrel{9}{\rightarrow} 0 & \text { tl: }[11] \stackrel{3}{\rightarrow} 1
\end{array}
$$

We consider resource boundedness of $\mathcal{R}_{1}$ with respect to the given (monomorphic) annotated signatures of Example 3. For simplicity we restrict to boundedness of enq. We leave it to the reader to check the other cases. In addition to the annotations for constructor symbols (cf. Example 1) we can always assume the presence of zero-cost annotations, e.g. $\sharp:[0 \times 0] \stackrel{0}{\rightarrow} 0$. Observe that Rule 6 frees the context $n: 15$ and cost 15 . 
Thus, we obtain the following derivation.

$$
\frac{\frac{\text { snoc: }[7 \times 0] \stackrel{14}{\rightarrow} 7}{q: 7, m:\left.0\right|^{14} \operatorname{snoc}(q, m): 7}(\text { app }) \frac{\text { enq: }[15] \stackrel{12}{\longrightarrow} 7}{n_{2}:\left.0\right|^{0} n_{2}: 0}(\text { var }) \frac{n_{1}:\left.15\right|^{12} \text { enq }\left(n_{1}\right): 7}{n_{1}: 15, n_{2}:\left.0\right|^{26}} \operatorname{snoc}\left(\text { enq }\left(n_{1}\right), n_{2}\right): 7}{\left.n:\left.15\right|^{26} \operatorname{spp} \text { (share }\right)} \text { (comp) }
$$

In comparison to [8, Example 13], where the annotations were found manually, we note that the use of the interleaving operation [8] has been avoided. This is due to the more general class of annotations considered in our prototype implementation (see Section 5).

The footprint relation forms a restriction of the judgement $\vdash$ without the use of weakening. Hence the footprint allows a precise control of the resources stored in the substitutions, as indicated by the next lemma.

Lemma 3. Let t be a normal form w.r.t. $\mathcal{R}$, where $t$ consists of constructor or constructorlike symbols only. If $\Gamma \mid \frac{k}{\mathrm{fp}} t: \vec{q}$, then $\Phi(t \sigma: \vec{q})=\Phi(\sigma: \Gamma)+k$.

Proof. Let $\Pi$ denote the derivation of the footprint $\Gamma \mid \frac{k}{f \mathrm{p}} t: \vec{q}$ and let $t=f\left(t_{1}, \ldots, t_{n}\right)$. We proceed by induction on $\Pi$. We restrict our attention to the cases where $\Pi$ amounts to a rule application or ends in a comp rule. The other cases are treated similarly.

Suppose $\Pi$ has the following form, so that $t=f\left(x_{1}, \ldots, x_{n}\right)$.

$$
\frac{f \in \mathcal{C} \cup \mathcal{D} \quad\left[\vec{p}_{1} \times \cdots \times \vec{p}_{n}\right] \stackrel{k}{\rightarrow} \vec{q} \in \mathcal{F}(f)}{x_{1}: \vec{p}_{1}, \ldots, x_{n}:\left.\vec{p}_{n}\right|^{k} f\left(x_{1}, \ldots, x_{n}\right): \vec{q}} .
$$

By assumption the annotation $f:\left[\vec{p}_{1} \times \cdots \times \vec{p}_{n}\right] \stackrel{k}{\rightarrow} \vec{q}$ is unique. Hence, we obtain:

$$
\Phi\left(f\left(x_{1}, \ldots, x_{n}\right) \sigma: \vec{q}\right)=k+\Phi\left(x_{1} \sigma: \vec{p}_{1}\right)+\cdots+\Phi\left(x_{n} \sigma: \vec{p}_{n}\right)=\Phi(\sigma: \Gamma)+k,
$$

from which the claim follows.

Suppose $\Pi$ ends in a comp rule and thus has the following form.

$$
\frac{\left.\overbrace{x_{1}: \vec{p}_{1}, \ldots, x_{n}: \vec{p}_{n}}^{=: \Delta}\right|^{k_{0}} f\left(x_{1}, \ldots, x_{n}\right): \vec{q} \quad \Gamma_{i} \aleph^{k_{i}} t_{i}: \vec{p}_{i} \quad \text { for all } i=1, \ldots, n}{\Gamma_{1}, \ldots, \Gamma_{n} \models^{k} f\left(t_{1}, \ldots, t_{n}\right): \vec{q}} .
$$

Wlog. $t$ is linear. By induction hypothesis we have for all $i=1, \ldots, n$ : $\Phi\left(t_{i} \sigma: \vec{p}_{i}\right)=$ $\Phi\left(\sigma: \Gamma_{i}\right)+k_{i}$. We set $\rho:=\left\{x_{i} \mapsto t_{i} \sigma \mid i=1, \ldots, n\right\}$. Again by induction hypothesis we conclude that $\Phi\left(f\left(x_{1}, \ldots, x_{n}\right) \rho: \vec{q}\right)=\Phi(\rho: \Delta)+k_{0}$. Now the claim follows as (i) $\Phi(t \sigma: \vec{q})=$ $\Phi\left(f\left(x_{1}, \ldots, x_{n}\right) \rho: \vec{q}\right)$, (ii) $\Phi(\rho: \Delta)=\sum_{i=1}^{n}\left(\Phi\left(\sigma: \Gamma_{i}\right)+k_{i}\right)$ and (iii) $k=\sum_{i=0}^{n} k_{i}$.

We state the following substitution lemma. The lemma follows by simple induction on $t$. 
Lemma 4. Let $\Gamma$ be a context and let $\sigma$ be a substitution consistent with $\Gamma$. Then $\Gamma \vdash t: \vec{q}$ implies $\vdash t \sigma: \vec{q}$.

We establish soundness with respect to relative innermost rewriting.

Theorem 5. Let $\mathcal{R} / \mathcal{S}$ be a resource bounded TRS and let $\sigma$ be a normalised such that $\sigma$ is consistent with the context $\Gamma$. Suppose $\left.\Gamma\right|^{k} t: \vec{p}$ and $t \sigma \stackrel{i}{\rightarrow} \mathcal{R}_{\mathcal{R}}^{K} u \tau, K \in\{0,1\}$ for a normalising substitution $\tau$. Then there exists a context $\Delta$ such that $\Delta \ell^{\ell} u: \vec{q}$ is derivable and $\Phi(\sigma: \Gamma)+k-\Phi(\tau: \Delta)-\ell \geqslant K$.

Proof. Let $\Pi$ denote the derivation of the judgement $\Gamma \mid k t: \vec{q}$. The proof proceeds by case distinction on derivation $D: t \sigma \stackrel{i}{\rightarrow}{ }_{\mathcal{R}}^{K} u \tau$ and side-induction on $\Pi$.

Suppose $D$ is empty, that is, $t \sigma$ is a normal form wrt. $\mathcal{R} \cup \mathcal{S}$. We distinguish two subcases. Either (i) $t \sigma$ contains only constructor or constructor-like symbols or (ii) $t \sigma$ contains at least one defined function symbol which does not occur as argument of the left-hand side of a rule in $\mathcal{R} \cup \mathcal{S}$.

For subcase (i), it suffices to show that $\Phi(\sigma: \Gamma)+k \geqslant \Phi(v: \vec{q})$ holds even under the assumption that no weakening rules are applied in $\Pi$. However, due to Lemma2, $\Gamma \mid \frac{k}{\mathrm{fp}} t: \vec{q}$ implies that $\Phi(\sigma: \Gamma)+k=\Phi(v: \vec{q})$. Thus the theorem follows. Now consider sub-case (ii). By definition $\Phi(v: \vec{q})=0$ and the theorem follows as potentials and amortised costs are non-negative.

Now suppose $D: t \sigma \stackrel{\mathrm{i}}{\rightarrow}_{\mathcal{R} / \mathcal{S}} u \tau$, that is $D$ is non-empty. We exemplify the proof on three subcases.

For subcase (i), we assume that $\Pi$ has the following form.

$$
\frac{f \in \mathcal{C} \cup \mathcal{D}\left[\vec{p}_{1} \times \cdots \times \vec{p}_{n}\right] \stackrel{k}{\rightarrow} \vec{q} \in \mathcal{F}(f)}{\left.\underbrace{x_{1}: \vec{p}_{1}, \ldots, x_{n}: \vec{p}_{n}}_{=\Gamma}\right|^{k} f\left(x_{1}, \ldots, x_{n}\right): \vec{q}} .
$$

Then $\sigma=\left\{x_{i} \mapsto v_{i} \mid i=1, \ldots, n\right\}$, where $v_{i} \in \mathrm{NF}$. By assumption on $D$ there exists a rule $f\left(l_{1}, \ldots, l_{n}\right) \rightarrow r$ and a normalised substitution $\tau$ such that $f\left(l_{1}, \ldots, l_{n}\right) \tau=t \sigma$ and $t \tau=u$. Wlog. $f\left(l_{1}, \ldots, l_{n}\right) \in \mathcal{R}$. As $\mathcal{R} / \mathcal{S}$ is bounded there exist variables $y_{1}, \ldots, y_{m}$, resource annotation $\vec{r}_{1}, \ldots, \vec{r}_{m}$ and an amortised costs $\ell$ such that the following judgement is derivable.

$$
\left.\overbrace{y_{1}: \vec{r}_{1}, \ldots, y_{l}: \vec{r}_{l}}^{=: \Delta}\right|^{k+\ell-1} r: \vec{q} .
$$

Due to Lemmata 2 and 3 we obtain $\Phi(\sigma: \Gamma)+k=\Phi(\tau: \Delta)+\ell$. The theorem follows.

For subcase (ii), we assume that $\Pi$ has the following form.

$$
\frac{\left.\overbrace{x_{1}: \vec{p}_{1}, \ldots, x_{n}: \vec{p}_{n}}^{=: \Delta_{0}}\right|^{k_{0}} f\left(x_{1}, \ldots, x_{n}\right): \vec{p} \quad \Gamma_{i} \mid \frac{k_{i}}{t_{i}: \vec{p}_{i}} \quad \text { for all } i=1, \ldots, n}{\Gamma_{1}, \ldots, \Gamma_{n}|k| k\left(t_{1}, \ldots, t_{n}\right): \vec{p}} .
$$

Wlog. $t$ is linear. As $t \sigma \stackrel{\text { i }}{\rightarrow} \mathcal{R} / \mathcal{S} u \tau$, there exist (potentially empty) subderivations $D_{i}: t_{i} \sigma \stackrel{\mathrm{i}}{\rightarrow}_{\mathcal{R} / \mathcal{S}} u_{i} \tau$ for all $i=1, \ldots, n$. We set $D_{0}:=f\left(x_{1}, \ldots, x_{k}\right) \rho \stackrel{\mathrm{i}}{\rightarrow}_{\mathcal{R} / \mathcal{S}} u \tau$. 
By side induction hypothesis we conclude the existence of contexts $\Delta_{i}$ and annotations $\overrightarrow{q_{i}}$ such that $\Delta_{i} \stackrel{\ell_{i}}{\stackrel{1}{-}} u_{i}: \vec{q}_{i}$ and $\Phi\left(\sigma: \Gamma_{i}\right)+k_{i} \geqslant \Phi\left(\tau: \Delta_{i}\right)+\ell_{i}$. Further let $\rho:=\left\{x_{i} \mapsto u_{i} \mid i=\right.$ $1, \ldots, n\}$. Then again by induction hypothesis, there exists context $\Delta^{\prime}$ and annotation $\vec{q}^{\prime}$ such that $\Delta^{\prime} \ell^{\ell_{0}} u: \vec{q}^{\prime}$ is derivable and $\Phi(\rho: \Delta)+k_{0} \geqslant \Phi\left(\tau: \Delta^{\prime}\right)+\ell_{0}$. Observe that at most one of the inequalities in the potentials is strict. By distinguishing all possible subcases, the theorem follows.

For subcase (iii), we assume $\Pi$ has the following form.

$$
\frac{\Gamma, x: \vec{r}, y:\left.\vec{s}\right|^{k} t[x, y]: \vec{q} \quad \curlyvee(\vec{p} \mid \vec{r}, \vec{s}) \quad x, y \text { are fresh }}{\Gamma, z:\left.\vec{q}\right|^{k} t[z, z]: \vec{q}} .
$$

Then the theorem follows from the side induction hypothesis in conjunction with Lemma[1]

The next corollary is an immediate consequence of the theorem, highlighting the connection to similar soundness results in the literature.

Corollary 6. Let $\mathcal{R} / \mathcal{S}$ be a bounded TRS and let $\sigma$ be a normalising substitution consistent with the context $\Gamma$. Suppose $\left.\Gamma\right|^{k} t: \vec{q}$ and $D: t \sigma \stackrel{i}{\rightarrow} !_{\mathcal{R} / \mathcal{S}} v \in \mathrm{NF}$. Then (i) $\vdash v: \vec{q}$ and (ii) $\Phi(\sigma: \Gamma)-\Phi(v: \vec{q})+k \geqslant|D|$ hold.

The next theorem defines suitable constraints on the resource annotations to deduce polynomial innermost runtime from Theorem[5] Its proof follows the pattern of the proof of Theorem 14 in $[8]$.

Theorem 7. Suppose that for each constructor c with $\left[\vec{p}_{1} \times \cdots \times \vec{p}_{n}\right] \stackrel{k^{\prime}}{\rightarrow} \vec{q} \in \mathcal{F}(c)$, there exists $\vec{r}_{i} \in \mathcal{A}$ such that $\vec{p}_{i} \leqslant \vec{q}+\vec{r}_{i}$ where $\max \vec{r}_{i} \leqslant \max \vec{q}=: r$ and $k^{\prime} \leqslant r$ with $\left|\vec{r}_{i}\right|<|\vec{q}|=: k$. Then $\Phi(v: \vec{q}) \leqslant r|v|^{k}$, and thus the innermost runtime complexity of the TRS under investigation is in $\mathcal{O}\left(n^{k}\right)$.

Proof. We proceed by induction on $v$. Observe that if $k=0$ then $\Phi(v: \vec{q})=0$. Otherwise, we have

$$
\begin{aligned}
\Phi\left(c\left(v_{1}, \ldots, v_{n}\right): \vec{q}\right) & \leqslant r+\Phi\left(v_{1}: \vec{p}_{1}\right)+\cdots+\Phi\left(v_{n}: \vec{p}_{n}\right) \\
& \leqslant r+\Phi\left(v_{1}: \vec{q}+\vec{r}_{1}\right)+\cdots+\Phi\left(v_{n}: \vec{q}+\vec{r}_{n}\right) \\
& =r+\Phi\left(v_{1}: \vec{q}\right)+\Phi\left(v_{1}: \vec{r}_{1}\right)+\cdots+\Phi\left(v_{n}: \vec{q}\right)+\Phi\left(v_{n}: \vec{r}_{n}\right) \\
& \leqslant r\left(1+\left|v_{1}\right|^{k}+\left|v_{1}\right|^{k-1}+\cdots+\left|v_{n}\right|^{k}+\left|v_{n}\right|^{k-1}\right),
\end{aligned}
$$

where we have applied the induction hypothesis in conjunction with Lemma 1. The last expressed is bounded by $r\left(1+\left|v_{1}\right|+\cdots+\left|v_{n}\right|\right)^{k}=r|v|^{k}$ due to the multinomial theorem.

We note that our running example satisfies the premise of Theorem 7 Thus the linear bound on the innermost runtime complexity of the running example $\mathcal{R}_{1}$ follows. The next example clarifies that without further assumptions potentials are not restricted to polynomials.

Example 4. Consider that we annotate the constructors for natural numbers as 0: [] $\stackrel{0}{\rightarrow} \vec{p}$ and s: $[2 \vec{p}] \stackrel{p_{1}}{\longrightarrow} \vec{p}$, where $\vec{p}=\left(p_{1}, \ldots, p_{k}\right)$. We then have, for example, $\Phi(t: 1)=2^{v}-1$, where $v$ is the value represented by $t$. 


\section{Implementation}

In this section we describe the details of important implementation issues. The realisations of the presented method can be seen twofold. On one hand we have a standalone program which tries to directly annotate the given TRS. While on the other hand the integration into $\mathrm{T}^{\top}$ [11] uses relative rewriting. Clearly, as an integration into $\mathrm{T}^{\top} \mathrm{T}^{\text {was }}$ planned from the beginning, the language used for the implementation of the amortised resource analysis module is Haskel 2 .

The central idea of the implementation is the collection of all signatures and arising constraints occurring in the inference tree derivations. To guarantee resource boundedness further constraints are added such that uniqueness and superposition of constructors (cf. Section 4) is demanded and polynomial bounds on the runtime complexity are guaranteed (cf. Theorem 7).

Inference Tree Derivation and Resource Boundedness. To be able to apply the inference rules the expected root judgement of each rule is generated (as in Example 3) by the program and the inference rules of Figure 2 are applied. To gain determinism the inference rules are ordered in the following way. The share-rule has highest priority, followed by app, var, comp and $w_{4}$. In each step the first applicable rule is used while the remaining weakening rules $w_{1}, w_{2}$ and $w_{3}$ are integrated in the aforementioned ones. For each application of an inference rule the emerging constraints are collected.

To ensure monomorphic typing of function signatures we keep track of a list of signatures. It uses variables in lieu of actual vectors. For each signature occurrence of defined function symbols the system refers to the corresponding entry in the list of signatures. Therefore, for each defined function symbol only one signature is added to the list of signatures. If the function occurs multiple times, the same references are used. Unlike defined function symbols multiple signature declarations of constructors are allowed, and thus each occurrence adds one signature to the list.

For the integration into $\mathrm{T}^{\mathrm{T}} \mathrm{T}$ we utilise the relative rewriting formulation. Instead of requiring all strict rules to be resource bounded, we weaken this requirement to have at least one strict rule being actually resource bounded, while the other rules may be annotated cost-free resource bounded. The SMT solver chooses which rule will be resource bounded. Clearly, this eases the constraint problem which is given to the SMT solver.

Superposition of Constructors. Recall that constructor and constructor-like symbols $f$ must satisfy the superposition principle. Therefore, for each annotation $\left[\overrightarrow{p_{1}} \times \cdots \times \overrightarrow{p_{n}}\right] \stackrel{k}{\rightarrow}$ $\vec{q}$ of $f$ it must be ensured that there is no annotation $\left[\lambda \cdot \overrightarrow{p_{1}} \times \cdots \times \lambda \cdot \overrightarrow{p_{n}}\right] \stackrel{\lambda \cdot k}{\longrightarrow} \overrightarrow{q^{\prime}}$ with $\lambda \in \mathbb{Q}^{+}$and $q \neq \lambda \cdot q^{\prime}$ in the corresponding set of annotated signatures. Therefore, for every pair $\left(q, q^{\prime}\right)$ with $q^{\prime} \geqslant q$ and $q>0$ either for every $\lambda>0: q^{\prime} \neq \lambda \cdot q$ or if $q^{\prime}=\lambda \cdot q$ then the annotation must be of the form $\left[\lambda \cdot \overrightarrow{p_{1}} \times \cdots \times \lambda \cdot \overrightarrow{p_{n}}\right] \stackrel{\lambda \cdot k}{\longrightarrow} \lambda \vec{\cdot} \cdot q$.

A naive approach is adding corresponding constraints for every pair of return annotations of a constructor symbol. This leads to universal quantifiers due to the scalar multiplication, which however, are available as binders in modern SMT solvers [22]. Early experiments revealed their bad performance. Overcoming this issue using Farkas'

\footnotetext{
${ }^{2}$ See http://haskell.org/
} 


$$
\begin{aligned}
& {\left[\vec{p}_{1}^{c f} \times \cdots \times \vec{p}_{n}^{c f}\right] \stackrel{\vec{k}^{c f}}{\rightarrow} \vec{q}^{c f} \in \mathcal{F}^{c f}(f)} \\
& \frac{\left[\vec{p}_{1} \times \cdots \times \vec{p}_{n}\right] \stackrel{k}{\rightarrow} \vec{q} \in \mathcal{F}(f)}{x_{1}: \vec{p}_{1}, \ldots, x_{n}:\left.\vec{p}_{n}\right|^{k} f\left(x_{1}, \ldots, x_{n}\right): \vec{q}}
\end{aligned}
$$

Figure 3: Additional app Rule for Cost-Free Derivation, where $f \in \mathcal{C} \cup \mathcal{D}$.

Lemma 23] is not possible here. This is due to the fact that a scaling variable for each annotation in every pair of annotations has to be introduced. Note that the number of constraints increase exponentially by requiring constraints over pairs of annotations. Thus, we developed a heuristic of spanning up a vector space using unit vectors for the annotation of the return types for each constructor. Each annotated signature of such a symbol must be a linear combination of the base signatures.

Both methods, universal quantifiers and base signatures lead to non-linear constraint problems. However, these can be handled by some SMT solver 3 . Thus, in contrast to the techniques presented in [17, 18, 3], which restrict the potential function to predetermined data structures, like lists or binary trees, our method allows any kind of data structure to be annotated.

Example 5. For instance consider the base constructors $\sharp_{1}:[(0,0) \times(1,0)] \stackrel{1}{\rightarrow}(1,0)$ and $\sharp_{2}:[(0,0) \times(2,1)] \stackrel{1}{\rightarrow}(0,1)$ for a constructor $\sharp$. An actual instance of an annotated signature is $n_{1} \cdot \sharp_{1}+n_{2} \cdot \sharp_{2}$ with $n_{1}, n_{2} \in \mathbb{N}$. As the return types can be seen as unit vectors of a Cartesian coordinate system the superposition and uniqueness properties hold.

Cost-Free Function Symbols. Inspired by Hoffmann [17, p.93ff] we additionally implemented a cost-free inference tree derivation when searching for non-linear bounds. The idea is that for many non-tail recursive functions the freed potential must be the one of the original function call plus the potential that gets passed on.

The inference rules are extended by an additional app-rule, which separates the function signature into two parts, cf. Figure 3. On the left there are the monomorphic and cost-free signatures while on the right a cost-free part is added. For every application of the rule the newly generated cost-free signature annotation must be cost-free resource bounded, for this the cost-free type judgement indicated has to be derived for any rule $f\left(l_{1}, \ldots, l_{n}\right) \rightarrow r$ and freed context $y_{1}: \vec{r}_{1}, \ldots, y_{l}: \vec{r}_{l}$ and cost $\ell$. Thus, the new set of annotations for a defined function symbols $f$ is given by the following set, cf. [17, p. 93].

$$
\left\{\left[\vec{p}_{1}+\lambda \cdot \vec{p}_{1}^{c f} \times \cdots \times \vec{p}_{n}+\lambda \cdot \vec{p}_{n}^{c f}\right] \stackrel{k+\lambda \cdot \vec{k}^{c f}}{\longrightarrow} \vec{q}+\lambda \cdot \vec{q}^{c f} \mid \lambda \in \mathbb{Q}^{+}, \lambda \geqslant 0\right\} .
$$

The decision of which app rule is applied utilises the strongly connected component (SCC) of the call graph analysis as done in [17, p.93ff].

Suppose $f$ is the function being analysed. Whenever, a currently deriving function $g$, where $g$ is not constructor-like, resides in the SCC of $f$ and the current derivation is

${ }^{3} \mathrm{We}$ use the SMT Solvers z3 (https://github.com/Z3Prover/z3/wiki) and MiniSmt (http://cl-informatik.uibk.ac.at/software/minismt/). 


\begin{tabular}{|c|c|c|c|c|c|c|}
\hline Testbed: TPDB & $\mathrm{O}(1)$ & $\mathrm{O}\left(n^{1}\right)$ & $\mathrm{O}\left(n^{2}\right)$ & $\mathrm{O}\left(n^{3}\right)$ & $\mathrm{O}(n \geqslant 4)$ & Fail \\
\hline $\mathrm{T}_{\mathrm{C}}^{\mathrm{T}}$ with $\mathrm{ARA}$ & 44 & 211 & 71 & 27 & 10 & 648 \\
\hline $\mathrm{T}_{\mathrm{C}}^{\mathrm{T}}$ without ARA & 44 & 210 & 68 & 28 & 8 & 653 \\
\hline Standalone ARA & 14 & 105 & 30 & 0 & 0 & 862 \\
\hline Standalone ARA with CF & 14 & 105 & 46 & 8 & 0 & 838 \\
\hline AProVE & 53 & 321 & 127 & 35 & 12 & 463 \\
\hline $\mathrm{T}_{\mathrm{C}}^{\mathrm{T}}$ with $\mathrm{ARA}$ & 3.55 & 9.87 & 17.32 & 26.02 & 43.39 & 50.01 \\
\hline $\mathrm{T}_{\mathrm{C}}^{\top}$ without ARA & 3.13 & 9.90 & 14.48 & 24.85 & 40.46 & 49.79 \\
\hline Standalone ARA & 0.10 & 0.24 & 0.81 & 0.00 & 0.00 & 2.44 \\
\hline Standalone ARA with CF & 0.15 & 1.00 & 8.65 & 10.94 & 0 & 18.54 \\
\hline AProVE & 2.03 & 34.43 & 151.77 & 190.17 & 199.65 & 252.52 \\
\hline
\end{tabular}

Figure 4: Experimental evaluation of $\mathrm{T}_{\mathrm{C}}^{\top}$ with ARA, $\mathrm{T}^{\top}$ without ARA and standalone ARA with and without cost-free (CF) derivations on the TPDB for number of instances (top) and required time in seconds (bottom).

not the cost-free analysis of $f$, the cost-free application rule of Figure 3 is used. In all other cases, the original function application rule of Figure 2 is used.

We have experimented with this heuristic, by pruning the need of the SCC requirement, such that the cost-free inference rule for application is not just allowed when $g$ resides in the SCC of $f$, but also when $g$ is reachable from $f$ in the call graph. This adaption however, increased the constraint problem size tremendously on one hand which obviously resulted in longer execution times but on the other hand could only infer one new cubic example and move one example from a cubic polynomial upper bound to quadratic. This makes sense, as the motivation behind this extension lies in the restricted feasibility of monomorphically annotations of recursive calls.

The implementation of the cost-free derivations uses a new set of signatures for which defined function symbol and constructor symbol signatures can occur multiple times. However, as above for each inference tree derivation only a single instance of a signature for each defined function symbol may be used.

Alternative Implementation of the Superposition Principle (Heuristics). Similar to [17, 8] we integrated the additive shift $\triangleleft(\vec{p})$ and interleaving $\vec{p} \| \vec{q}$ for constructors when type information is given. Here $\triangleleft\left(p_{1}, \ldots, p_{k}\right):=\left(p_{1}+p_{2}, p_{2}+p_{3}, \ldots, p_{k-1}+p_{k}, p_{k}\right)$ and $\vec{p} \| \mid \vec{q}:=\left(p_{1}, q_{1}, p_{2}, q_{2}, \ldots, p_{k}, q_{k}\right)$, where the shorter of the two vectors is padded with $0 \mathrm{~s}$. These heuristics are designed such that the superposition principle holds, without the need of base annotations. Therefore, the constraint problem automatically becomes linear whenever these heuristics are used which tremendously reduces the execution times.

\section{Experimental Evaluation}

In this section we evaluate the prototype implementation of the amortised analysis and how it deals with some selected examples including the paper's running example queue. All experiment:4 were conducted on a machine with an Intel(R) Core(TM) i7-7700 CPU @ $3.60 \mathrm{GHz}$ and 16GB RAM. The timeout was set to 60 seconds. The evaluation is split in the core application of the prototype on first-order TRSs as well as the assessment of

${ }^{4}$ Detailed data is available at http://cl-informatik.uibk.ac.at/software/tct/experiments/ara_worstcase/. 


\begin{tabular}{l|cccccc} 
Testbed: TRS programs & $\mathrm{O}(1)$ & $\mathrm{O}\left(n^{1}\right)$ & $\mathrm{O}\left(n^{2}\right)$ & $\mathrm{O}\left(n^{3}\right)$ & $\mathrm{O}(n \geqslant 4)$ & Fail \\
\hline $\mathrm{T}^{\top}$ ' with ARA & 2 & 45 & 24 & 8 & 2 & 35 \\
$\mathrm{~T} \mathrm{C}^{\top}$ without ARA & 2 & 42 & 26 & 7 & 2 & 37 \\
Standalone ARA & 1 & 43 & 2 & 0 & 0 & 70 \\
Standalone ARA with CF & 1 & 43 & 14 & 1 & 0 & 57 \\
\hline $\mathrm{T}^{\top}$ with ARA & 0.05 & 1.92 & 10.10 & 27.20 & 51.65 & 15.11 \\
$\mathrm{~T}^{\top}$ without ARA & 0.02 & 1.75 & 8.81 & 21.83 & 42.76 & 15.58 \\
Standalone ARA & 0.04 & 0.23 & 0.14 & 0.00 & 0.00 & 0.25 \\
Standalone ARA with CF & 0.08 & 1.56 & 8.97 & 1.21 & 0.00 & 54.91 \\
\hline
\end{tabular}

Figure 5: Experimental evaluation of $\mathrm{T}^{\top}{ }^{\top}$ with $A R A, T_{C}^{\top}$ without ARA and standalone ARA with and without cost-free ( $\mathrm{CF}$ ) derivations on the TRS testbed for number of instances (top) and required time in seconds (bottom).

the combination of the provided prototype in connection with the HOCA transformation. The latter is able to verify the worst-case bounds of higher-order functional programs fully automatically.

Thus for benchmarking we first elaborated the amortised resource analysis (ARA) directly on the TRSs. As these tests directly evaluate the applicability and functionality of ARA for TRSs we provide results for two separate testbeds. First we utilize the runtime-complexity-innermost-rewriting folder of the TPDB5 which includes 1011 problems, and second a collection consisting of 116 TRSs representing first-order functional programs [24, 25], transformations from higher-order programs [12, 13], or RaML programs [18] and interesting examples from the TPDB. We will refer to the later as TRS testbed, while the former is called TBDB testbed for which we use as a comparison the results of AProVE6. For both testbeds we compared the competition version of $\mathrm{T}_{\mathrm{C}}^{\mathrm{T}}$ without the amortised resource analysis (ARA) to a version where we integrated ARA. The integration into $\mathrm{T}_{\mathrm{C}}^{\mathrm{T}}$ is configured to not use any cost-free derivations $(\mathrm{CF})$ as the toolchain of $\mathrm{T}^{\top}$ already simplifies the problems such that the additional constraints become an almost always useless overhead. Thus to provide insights in the cost-free derivation mechanism we compare the results of standalone ARA with and without the cost-free derivations as well.

Figure 4 presents the experimental evaluation on the TPDB testbed. As expected ARA can solve less examples than ${ }^{\top} C^{\top}$ which makes sense as it includes several techniques like polynomial interpretations, matrix interpretations [26] or dependency pairs [27, 28]. Nonetheless ARA, which is able to infer univariate bounds only, can solve a reasonable amount of examples. It can be seen that ARA with cost-free derivations can solve more examples in the quadratic and cubic case to the expense of increased running time. Recall that cost-free derivations are important for non-tail recursive functions. This extension to ARA increases the applicability of the analysis tremendously. For instance in the quadratic case the prototype is able to increase the solved instances by more than $50 \%$. However, also note that the increase of running time for ARA with cost-free derivations as compared to ARA without cost-free derivations increases non-linearly. This is due to the additional constraints introduced. As can be seen in the table AProVE is able to solve

\footnotetext{
${ }^{5} \mathrm{We}$ refer to Version 10.4 of the Termination Problem Database, available from http://cl2-informatik.uibk.ac.at/mercurial.cgi/TPDB

${ }^{6}$ See https://aprove-developers.github.io/trs_complexity_via_its/ for detailed results of AProVE. Timeout: 300 seconds, Intel Xeon with 4 cores at $2.33 \mathrm{GHz}$ and $16 \mathrm{~GB}$ of RAM.
} 
most instances, where the time required to solve the problems increases with increasing complexity classes.

When comparing $\mathrm{T}^{\top}$ with ARA and $\mathrm{T}^{\top} \mathrm{T}^{\mathrm{w}}$ without ARA one can see that 5 more examples can be solved. This includes examples from the RaML folder, like quicksort, bitvectors and depth/breadth first search. Furthermore, sometimes better bounds can be inferred with integrated ARA. For instance $T_{C} T$ without ARA infers a quadratic bound for the example \#3.42 which computes the binary representation of numbers, while with ARA the correct linear bound can be inferred. However, by adding this additional method to the toolchain of $\mathrm{T}^{\top} \mathrm{T}^{\top}$ we observed a slightly increased average running time. Nonetheless the actual running time is closely problem related. To exemplify, for the above mention program \#3.42 the running time decreases by almost a factor of 10 when ARA is added (from 21.45s to 2.61s), while for the example appendAll the running time increases by a factor of 2.5 (from $1.05 \mathrm{~s}$ to $2.72 \mathrm{~s}$ ).

Figure 5 provides the results of the experimental evaluation on the TRS testbed. Again the number of solved instances increases by allowing cost-free derivations as can be seen on the standalone ARA results. However, the running times increase as well. Furthermore, it can be seen that standalone ARA is able to infer more examples with linear worst-case bounds as $\mathrm{T}_{\mathrm{C}} \mathrm{T}$ without ARA. However, for higher polynomial bounds the toolchain of $T_{C}$ is required. As the impact of ARA is higher on this testbed we can see that the methods works best in the setting of program translations. $\mathrm{T}^{\top} \mathrm{T}$ with ARA can solve the examples bfs.raml and decrease as opposed to $\mathrm{T}_{\mathrm{C}} \mathrm{T}$ without ARA. Furthermore, better bounds for \#3.42 and queue can be found. In terms of running times we again observed a slight increase when ARA was added to ${ }^{\top} C^{\top}$.

For the second type of evaluation we collected combination of selected OCaml programs from both the HOCA [12] and the RaML testbed, complemented with interesting examples. Overall the testbed includes 48 programs. For this evaluation category we analyse the capabilities of ARA in connection with the HOCA transformation and compare the results to RaML, which uses an amortised analysis based on the OCaml code directly. The results of the evaluation are summarised in Figure 6. To provide further insights we tested the method in various combinations. First we used the tool TCT HOCA, which translates the input OCaml program to a TRS and then subsequently calls $\mathrm{T}_{\mathrm{C}} \mathrm{T}$. However, the type information from the OCaml code is lost during the translation process as the $\mathrm{T}^{\top}$ analysis in the current form is type-less. Again we evaluate the tool with and without the integration of ARA. Nonetheless, as the type information can be utilized by the heuristics we integrated the transformation process into ARA as well and compare the results of standalone ARA on the (pre-translated) TRS without type information (TRS) to the results of standalone ARA with the integration of the translation and thus with type information (HOCA). Finally we compare the results to the latest version of RaML (of July 2018). Recall that RaML is able to infer multivariate bounds, while ARA is built for univariate bounds only.

When evaluating the standalone ARA version we observed no difference in terms of solved instances. The only difference provided in the table is one additional solved instance for standalone ARA on the TRS. This is due to the reason that the integrated transformation takes more than 60 seconds (about 300s) and thus the tool runs into a timeout. Without timeout the same number of solved instances are gained. This makes sense, as the heuristics only reduce the constraints to a linear problem (LP), while not providing any strength. However, in most cases the running time decreases when type 


\begin{tabular}{l|cccccc} 
Testbed: HOCA & $\mathrm{O}(1)$ & $\mathrm{O}\left(n^{1}\right)$ & $\mathrm{O}\left(n^{2}\right)$ & $\mathrm{O}\left(n^{3}\right)$ & $\mathrm{O}\left(n^{\geqslant}\right)$ & Fail \\
\hline TC T HOCA with ARA $^{\top}$ H HOCA without ARA & 1 & 17 & 8 & 1 & 2 & 19 \\
TC & 1 & 17 & 7 & 2 & 2 & 19 \\
Standalone ARA CF (TRS) & 1 & 18 & 5 & 0 & 0 & 24 \\
Standalone ARA CF (HOCA) & 1 & 17 & 5 & 0 & 0 & 25 \\
Raml-1.4.1 & 1 & 12 & 7 & 8 & 1 & 19 \\
\hline TC HOCA with ARA & 0.09 & 1.53 & 7.00 & 8.04 & 35.81 & 57.28 \\
TC HOCA without ARA & 0.03 & 0.59 & 4.70 & 30.35 & 5.34 & 57.21 \\
Standalone ARA CF (TRS) & 0.10 & 0.38 & 49.02 & 0.00 & 0.00 & 46.02 \\
Standalone ARA CF (HOCA) & 0.07 & 0.78 & 39.50 & 0.00 & 0.00 & 46.66 \\
Raml-1.4.1 & 0.03 & 0.07 & 0.79 & 1.10 & 3.38 & 12.12 \\
\hline
\end{tabular}

Figure 6: Experimental evaluation of $\mathrm{T}_{\mathrm{C}}^{\mathrm{T}}$ HOCA with ARA, TCT HOCA without ARA and standalone ARA with cost-free (CF) derivations on the translated TRS (without type information) and directly on the OCaml code which calls the HOCA transformation. The tools were evaluated on the HOCA testbed for instances (top) and required time in seconds (bottom).

information is available as the constraint problem can be solved without backtracking mechanism. This can be seen best in the column for quadratically inferred worst-case bounds, where the running time decreases by almost 10s. Furthermore, as ARA is based on base vectors it always tries to improve the quality of the solution by using non-linear constraint solving while RaML relies on heuristics only. This explains the huge gap of running time between RaML and standalone ARA. Finally when looking at the $\mathrm{T}_{\mathrm{C}} \mathrm{T}$ HOCA results we observe that the version with ARA can find a better bound for the example calculator.raml. Furthermore, for this testbed $\mathrm{T}_{\mathrm{C}}^{\top} \mathrm{HOCA}$ provides comparable results to RaML. However, the solved instances are different to a great extend. For instance, while all tools but RaML are able to solve examples like avanzini_original.hoca, btree_lookup.hoca, or flip.hoca, only RaML is able to provide bounds for 13 examples including quicksort.raml, bigints_add.raml, or isort.raml (see detailed results). These results are due to several reasons. First RaML does not support the user-defined type in btree_lookup.hoca as discussed above. Then RaML does not support lazy evaluation, while the HOCA transformation does. Third, for large programs the transformation process of $\mathrm{T}_{\mathrm{C}} \mathrm{T}$ HOCA can take long which results in the fact that either no or only little time is left for the analysis. In the following we will investigate some of the programs in detail.

\#3.42 - Binary representation. Given a number $n$ in unary encoding as input, the TRS computes the binary representation $(n)_{2}$ by repeatedly halving $n$ and computing the last bit, see the Appendix for the TRS. The optimal runtime complexity of $\mathcal{R}_{1}$ is linear in $n$. For this, first observe that the evaluation of half $\left(\mathrm{s}^{m}(0)\right)$ and lastbit $\left(\mathrm{s}^{m}(0)\right)$ requires about $m$ steps in total. Secondly, $n$ is halved in each iteration and thus the number of steps can be estimated by $\sum_{i=0}^{k} 2^{i}$, where $k:=\left|(n)_{2}\right|$. As the geometric sum computes to $2 \cdot 2^{k}-1$, the claim follows. Such a precise analysis is enabled by an amortised analysis, which takes the sequence of subsequent function calls and their respective arguments into account. Compared to former versions of $\mathrm{T}_{\mathrm{C}}^{\mathrm{T}}$ which reported $\mathrm{O}\left(n^{2}\right)$ we find this optimal linear bound of $\mathrm{O}(n)$ when ARA is enabled. Furthermore, the best case analysis of ARA shows that this bound is tight by returning $\Omega(n)$. Similarly AProVE [29] yields the tight bound employing a size abstraction to integer transition systems (ITSs for short), cf. [30]. The resulting ITSs are then solved with CoFloCo [31], which also embodies an amortisation analysis. 


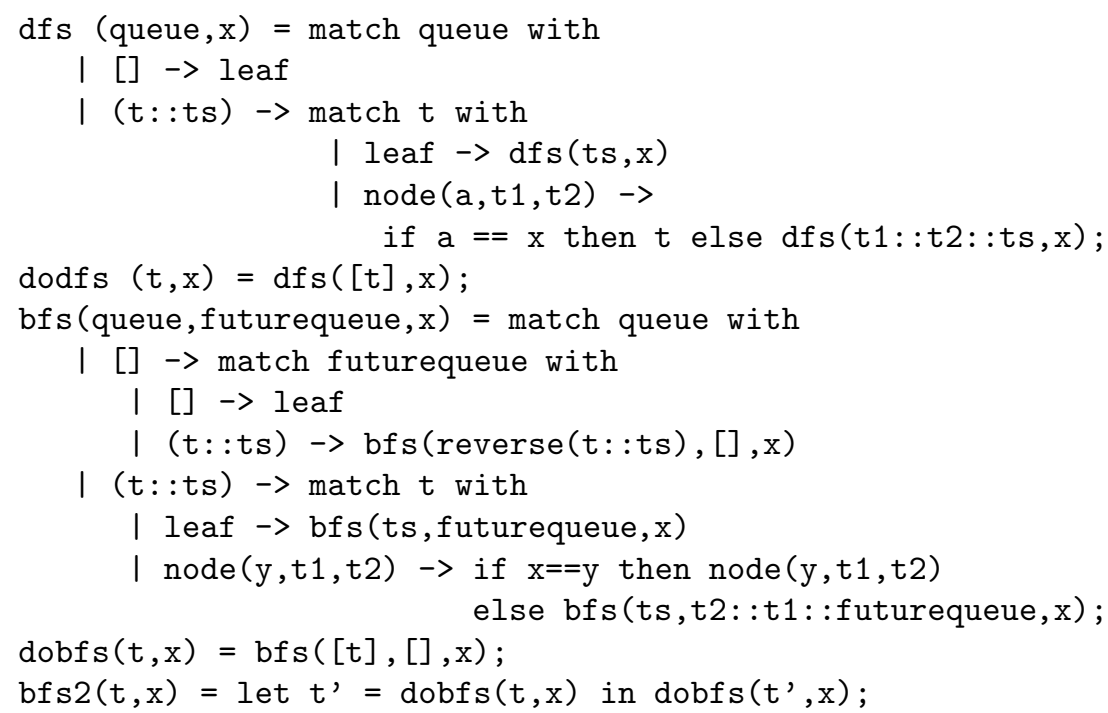

Figure 7: DFS and BFS in RaML Syntax [17, p.70] of which the translation to an TRS can be found in the TPDB.

insertionsort.raml. Insertionsort from the TPDB has quadratic runtime complexity. ARA with cost-free derivations enabled is able to infer this bound. Similarly can TCT with ARA using the default setup, while when looking for the best bound only it is unable to handle the trade off between execution time and tightness of the bound and runs into a timeout. AProVE infers $\mathrm{O}\left(n^{2}\right)$ as well. This bound is tight [17, p.158ff]. The best case analysis finds a linear lower bound for this implementation of insertionsort.

tpa2 - Multiple Subtraction. This TRS from the TPDB iterates subtraction until no more rules can be applied. The latest version of $T_{C} T$ with ARA is in contrast to $T_{C} T^{T}$ without ARA able to solve the problem. The inferred quadratic worst-case bound coincides with the bounds provided by AProVE.

bfs.raml - Depth/Breadth-First Search. This program, found in the TPDB, is a translation of depth-first search (DFS) and breadth-first search (BFS) from RaML syntax, see Figure 7 for the RaML code. Note that the TRS uses strict rules for the equality check which recurses on the given data structure. In DFS a binary tree is searched one branch after the other for a matching entry while BFS uses two lists to keep track of nodes of a binary tree to be visited. The first one is used to traverse on the nodes of the current depth, whereas the second list collects all nodes of the next depth to visit. After each iteration the futurelist is reversed. Further, note that BFS is called twice in the function bfs2. Standalone ARA and $\mathrm{T}_{C} \mathrm{~T}$ with ARA are the only tools which are able to infer a complexity bound of $\mathrm{O}\left(n^{2}\right)$. 


\section{Conclusion}

In this paper we have established a novel automated amortised cost analysis for term rewriting. In doing so we have not only implemented the methods detailed in earlier work [8], but also generalised the theoretical basis considerably. We have provided a prototype implementation and integrated into $\mathrm{T}_{\mathrm{C}} \mathrm{T}$.

More precisely, we have extended the method of amortised resource analysis to unrestricted term rewrite systems, thus overcoming typical restrictions of functional programs like left-linearity, pattern based, non-ambiguity, etc. This extension is non-trivial and generalises earlier results in the literature. Furthermore, we have lifted the method to relative rewriting. The latter is the prerequisite to a modular resource analysis, which we have provided through the integration into $\mathrm{T}_{\mathrm{C}} \mathrm{T}$. The provided integration of amortised resource analysis into $\mathrm{T}_{\mathrm{C}} \mathrm{T}$ has led to an increase in overall strength of the tool (in comparison to the latest version without ARA and the current version of AProVE). Furthermore in a significant amount of cases we could find better bounds than before.

In future work we want to focus on lifting the provided amortised analysis in two ways. First we want to extend the provided univariate analysis to a multivariate analysis akin the analysis provided in RaML. The theoretical foundation for this has already been provided by Hofmann et al. [9]. However efficient automation of the method proposed in [9] requires some sophistication. Secondly, we aim to overcome the restriction to constant amortised analysis and provide an automated (or at least automatable) method establishing logarithmic amortised analysis. This aims at closing the significant gap of existing methods in contrast to the origin of amortised analysis [1, 2], compare also [32].

\section{References}

[1] D. Sleator, R. Tarjan, Self-adjusting binary trees, in: Proc. of the 15th STOC, ACM, 1983, pp. 235-245. doi:10.1145/800061.808752.

[2] R. Tarjan, Amortized computational complexity, SIAM J. Alg. Disc. Meth 6 (2) (1985) $306-318$.

[3] J. Hoffmann, A. Das, S. Weng, Towards automatic resource bound analysis for OCaml, in: Proc. 44th POPL, ACM, 2017, pp. 359-373.

[4] E. Albert, P. Arenas, S. Genaim, G. Puebla, Closed-form upper bounds in static cost analysis, JAR $46(2)$.

[5] A. Flores-Montoya, Upper and lower amortized cost bounds of programs expressed as cost relations, in: Proc. 21st FM, Vol. 9995 of LNCS, 2016, pp. 254-273. doi:10.1007/978-3-319-48989-6_16

[6] M. Sinn, F. Zuleger, H. Veith, A simple and scalable static analysis for bound analysis and amortized complexity analysis, in: Proc. Software Engineering, Vol. 252 of LNI, 2016, pp. 101-102.

[7] C. Okasaki, Purely functional data structures, Cambridge University Press, 1999.

[8] M. Hofmann, G. Moser, Amortised resource analysis and typed polynomial interpretations, in: Proc. of Joint 25th RTA and 12th TLCA, Vol. 8560 of LNCS, 2014, pp. 272-286.

[9] M. Hofmann, G. Moser, Multivariate amortised resource analysis for term rewrite systems, in: Proc. 13th TLCA, Vol. 38 of LIPIcs, 2015, pp. 241-256. doi:10.4230/LIPIcs.TLCA.2015.241

[10] M. Avanzini, U. D. Lago, Automating sized-type inference for complexity analysis, PACMPL 1 (ICFP) (2017) 43:1-43:29. doi:10.1145/3110287

[11] M. Avanzini, G. Moser, M. Schaper, TcT: Tyrolean Complexity Tool, in: Proc. 22nd TACAS, LNCS, 2016, pp. 407-423.

[12] M. Avanzini, U. D. Lago, G. Moser, Analysing the complexity of functional programs: higher-order meets first-order, in: Proc. 20th ICFP, ACM, 2015, pp. 152-164. doi:10.1145/2784731.2784753

[13] M. Avanzini, U. D. Lago, G. Moser, Analysing the complexity of functional programs: Higher-order meets first-order (long version), CoRR abs/1506.05043, submitted.

[14] G. Moser, M. Schneckenreither, Amortised analysis for bestcase lowerbounds, in preparation (2018).

[15] G. Moser, M. Schneckenreither, Automated amortised resource analysis for term rewrite systems, in: Proc. 14th FLOPS, Vol. 10818 of LNCS, 2018, pp. 214-229. doi:10.1007/978-3-319-90686-7\_14 
[16] G. Moser, Uniform resource analysis by rewriting: Strenghts and weaknesses (invited talk), in: In Proc. 2nd FSCD, Vol. 84 of LIPIcs, 2017, pp. 2:1-2:10.

[17] J. Hoffmann, Types with potential: Polynomial resource bounds via automatic amortized analysis, Ph.D. thesis, Ludwig-Maximilians-Universität München (2011).

[18] J. Hoffmann, K. Aehlig, M. Hofmann, Multivariate amortized resource analysis, TOPLAS 34 (3) (2012) 14.

[19] F. Baader, T. Nipkow, Term Rewriting and All That, Cambridge University Press, 1998.

[20] TeReSe, Term Rewriting Systems, Vol. 55 of Cambridge Tracks in Theoretical Computer Science, Cambridge University Press, 2003.

[21] R. Thiemann, The DP framework for proving termination of term rewriting, Ph.D. thesis, University of Aachen, Department of Computer Science (2007).

[22] C. Barrett, P. Fontaine, C. Tinelli, The SMT-LIB Standard: Version 2.6, Tech. rep., Department of Computer Science, The University of Iowa, available at www.SMT-LIB.org (2017).

[23] J. Farkas, Theorie der einfachen Ungleichungen., Journal für die reine und angewandte Mathematik $124(1902) 1-27$

[24] A. Glenstrup, Terminator ii: Stopping partial evaluation of fully recursive programs, Master's thesis, DIKU, technical Report DIKU-TR-99/8 (1999).

[25] C. Frederiksen, Automatic runtime analysis for first order functional programs, Master's thesis, DIKU, dIKU TOPPS D-470 (2002)

[26] G. Moser, A. Schnabl, J. Waldmann, Complexity analysis of term rewriting based on matrix and context dependent interpretations, in: Proc. 28th IARCS Annual Conference on Foundations of Software Technology and Theoretical Computer Science, Vol. 2 of LIPIcs, 2008, pp. 304-315.

[27] N. Hirokawa, G. Moser, Automated complexity analysis based on the dependency pair method, in: Proc. 4th IJCAR, no. 5195 in LNAI, 2008, pp. 364-380.

[28] T. Arts, J. Giesl, Termination of term rewriting using dependency pairs, Theoretical Computer Science 236 (1-2) (2000) 133-178.

[29] J. Giesl, C. Aschermann, M. Brockschmidt, F. Emmes, F. Frohn, C. Fuhs, J. Hensel, C. Otto, M. Plücker, P. Schneider-Kamp, T. Ströder, S. Swiderski, R. Thiemann, Analyzing program termination and complexity automatically with aprove, JAR 58 (1) (2017) 3-31. doi:10.1007/s10817-016-9388-y

[30] M. Naaf, F. Frohn, M. Brockschmidt, C. Fuhs, J. Giesl, Complexity analysis for term rewriting by integer transition systems, in: International Symposium on Frontiers of Combining Systems, Springer, 2017, pp. 132-150.

[31] A. Flores-Montoya, Cofloco: System description, in: 15th International Workshop on Termination, 2016 , p. 20

[32] T. Nipkow, Amortized complexity verified, in: Proc. 6th ITP, Vol. 9236 of LNCS, 2015, pp. 310-324. doi:10.1007/978-3-319-22102-1_21 